PROCEEDINGS OF THE

AMERICAN MATHEMATICAL SOCIETY

Volume 133, Number 2, Pages 473-480

S 0002-9939(04)07538-0

Article electronically published on September 2, 2004

\title{
CONVERGENCE OF PATHS AND APPROXIMATION OF FIXED POINTS OF ASYMPTOTICALLY NONEXPANSIVE MAPPINGS
}

\author{
C. E. CHIDUME, JINLU LI, AND A. UDOMENE
}

(Communicated by Joseph A. Ball)

\begin{abstract}
Let $E$ be a real Banach space with a uniformly Gâteaux differentiable norm possessing uniform normal structure, $K$ be a nonempty closed convex and bounded subset of $E, T: K \longrightarrow K$ be an asymptotically nonexpansive mapping with sequence $\left\{k_{n}\right\}_{n} \subset[1, \infty)$. Let $u \in K$ be fixed, $\left\{t_{n}\right\}_{n} \subset(0,1)$ be such that $\lim _{n \rightarrow \infty} t_{n}=1, t_{n} k_{n}<1$, and $\lim _{n \rightarrow \infty} \frac{k_{n}-1}{k_{n}-t_{n}}=0$. Define the sequence $\left\{z_{n}\right\}_{n}$ iteratively by $z_{0} \in K, z_{n+1}=\left(1-\frac{t_{n}}{k_{n}}\right) u+\frac{t_{n}}{k_{n}} T^{n} z_{n}, n=0,1,2, \ldots$. It is proved that, for each integer $n \geq 0$, there is a unique $x_{n} \in K$ such that $x_{n}=\left(1-\frac{t_{n}}{k_{n}}\right) u+\frac{t_{n}}{k_{n}} T^{n} x_{n}$. If, in addition, $\lim _{n \rightarrow \infty}\left\|x_{n}-T x_{n}\right\|=0$ and $\lim _{n \rightarrow \infty}\left\|z_{n}-T z_{n}\right\|=0$, then $\left\{z_{n}\right\}_{n}$ converges strongly to a fixed point of $T$.
\end{abstract}

\section{INTRODUCTION}

Let $E$ be a normed linear space with dual $E^{*}$, and let $K$ be a nonempty subset of $E$. Let $J: E \longrightarrow 2^{E^{*}}$ denote the normalized duality mapping defined by $J(x):=$ $\left\{f \in E^{*}:\langle x, f\rangle=\|x\|^{2},\|f\|=\|x\|, x \in E\right\}$. A mapping $T: K \longrightarrow K$ is called uniformly L-Lipschitzian $(L>0)$ if $\left\|T^{n} x-T^{n} y\right\| \leq L\|x-y\|, \forall x, y \in K$ and for all integers $n \geq 0$. The mapping $T$ is called asymptotically nonexpansive if there exists a sequence $\left\{k_{n}\right\} \subset[1, \infty)$ with $\lim _{n \rightarrow \infty} k_{n}=1$ such that $\left\|T^{n} x-T^{n} y\right\| \leq$ $k_{n}\|x-y\|$ for all integers $n \geq 0$. It is clear that every asymptotically nonexpansive mapping is uniformly $L$-Lipschitzian for some constant $L>0$. The asymptotically nonexpansive mappings are important generalizations of nonexpansive mappings, where a mapping $T$ is said to be nonexpansive if $\|T x-T y\| \leq\|x-y\|$ for all $x, y \in E$.

The asymptotically nonexpansive mappings were introduced by Goebel and Kirk [7], and they proved that if $K$ is a nonempty bounded closed and convex subset of a uniformly convex Banach space $E$, and $T: K \longrightarrow K$ is asymptotically nonexpansive, then $T$ has a fixed point. In [8], they extended this result to the broader class of uniformly $L$-Lipschitzian mappings with $L<\gamma$, where $\gamma$ is sufficiently near 1 . Several authors have investigated iterative methods for approximating fixed points of asymptotically nonexpansive mappings (e.g., Bruck, Kuczumow and Reich [3],

Received by the editors June 12, 2003 and, in revised form, October 6, 2003.

2000 Mathematics Subject Classification. Primary 47H06, 47H09, 47J05, 47J25.

Key words and phrases. Asymptotically nonexpansive mappings, fixed points, uniformly Lipschitzian mappings. 
Chang 4, Lim and Xu [11, Schu [14, 15], Shioji and Takahashi [12, 13], Tan and $\mathrm{Xu}$ [16]). In [15], Schu proved the following theorems.

Theorem S1 ([15]). Let (E,\|.\|) be a smooth reflexive Banach space possessing a duality mapping $J: E \longrightarrow E^{*}$ that is weakly sequentially continuous at $0 ; \emptyset \neq A \subset$ $E$ closed, bounded and star-shaped with respect to $0 ; T: A \longrightarrow A$ asymptotically nonexpansive with sequence $\left\{k_{n}\right\}_{n} \subset[1, \infty)$ and uniformly asymptotically regular (see 15] for definition), $(i d-T)$ demiclosed; $\left\{\lambda_{n}\right\}_{n} \subset\left(\frac{1}{2}, 1\right) ; \lim _{n \rightarrow \infty} \lambda_{n}=1 ; k_{n} \leq$ $\frac{\lambda_{n}^{2}}{2 \lambda_{n}-1}$ for all integers $n \geq 0$. Then,

(i) for each $n \in \mathbb{N}$ there is exactly one $x_{n} \in A$ such that $x_{n}=\frac{\lambda_{n}}{k_{n}} T^{n} x_{n}$,

(ii) $\left\{x_{n}\right\}_{n}$ converges strongly to some fixed point of $T$.

Theorem S2 ([15]). Let (E, $\|\|$.$) be a normed space; \emptyset \neq A \subset E$ bounded and starshaped with respect to $0 ; T: A \longrightarrow A$ asymptotically nonexpansive with sequence $\left\{k_{n}\right\}_{n} \subset[1, \infty) ;\left\{\lambda_{n}\right\}_{n} \subset(0,1)$ with $\lim _{n \rightarrow \infty} \lambda_{n}=1 ;\left\{x_{n}\right\}_{n} \subset A ; \mu_{n}=\frac{\lambda_{n}}{k_{n}}$ for all integers $n \geq 0, z_{0} \in A ; x_{n}=\frac{\lambda_{n}}{k_{n}} T^{n} x_{n}$ for all integers $n \geq 0$, and $z_{n+1}:=\frac{\lambda_{n+1}}{k_{n+1}} T^{n} z_{n}$ for all integers $n \geq 0 ;\left\{\epsilon_{n}\right\}_{n} \subset(0, \infty)$ such that $\left(\left\{\epsilon_{n}\right\}_{n},\left\{\mu_{n}\right\}_{n}\right)$ is admissible (see, e.g., [15] or [10] for a definition). Also

(i) $\left\{\frac{1-\mu_{n}}{1-\lambda_{n}}\right\}$ is bounded;

(ii) $\left\|T^{n} x-T^{n+1} x\right\| \leq \epsilon_{n}$ for all integers $n \geq 0$ and all $x \in A$.

Assume further that $q:=\lim _{n \rightarrow \infty} x_{n}$ exists. Then $\lim _{n \rightarrow \infty} z_{n}=q$.

Theorem S1 provides sufficient conditions for the path given by the sequence $\left\{x_{n}\right\}_{n}$ for an asymptotically nonexpansive mapping to converge strongly to a fixed point of $T$. However, since $J$ is assumed to be weakly sequentially continuous at 0 , the theorem is not applicable to $L_{p}$ spaces $(p>1, p \neq 2)$. In [11], Lim and Xu proved a path-convergence theorem for asymptotically nonexpansive mappings in the more general uniformly smooth Banach spaces. These spaces include the $L_{p}$ spaces, $1<p<\infty$. They proved the following theorem.

Theorem LX ([11, Theorem 2, p. 1348]). Let E be a uniformly smooth Banach space, $K$ a nonempty closed convex and bounded subset of $E, T: K \longrightarrow K$ an asymptotically nonexpansive mapping with sequence $\left\{k_{n}\right\}_{n} \subset[1, \infty)$. Fix $u \in K$ and let $\left\{t_{n}\right\}_{n} \subset[0,1)$ be chosen such that $\lim _{n \rightarrow \infty} t_{n}=1$ and $\lim _{n \rightarrow \infty} \frac{k_{n}-1}{k_{n}-t_{n}}=0$. Then,

(i) for each integer $n \geq 0$, there is a unique $x_{n} \in K$ such that

$$
x_{n}=\left(1-\frac{t_{n}}{k_{n}}\right) u+\frac{t_{n}}{k_{n}} T^{n} x_{n} .
$$

Suppose, in addition, that $\lim _{n \rightarrow \infty}\left\|x_{n}-T x_{n}\right\|=0$. Then,

(ii) the sequence $\left\{x_{n}\right\}_{n}$ converges strongly to a fixed point of $T$.

Very recently $\mathrm{Xu}$ [17] proved the following theorem (which is the main result of [17]) for iterative approximation of a fixed point of a nonexpansive mapping in uniformly smooth Banach spaces.

Theorem Xu ([17, Theorem 3.1, p. 244]). Let X be a uniformly smooth Banach space, $C$ be a closed convex subset of $X$, and $S: C \longrightarrow C$ be a nonexpansive mapping with a fixed point. Let $u, x_{0} \in C$ be given. Assume that $\left\{\alpha_{n}\right\} \subset[0,1]$ 
satisfies the following conditions:

(i) $\lim _{n \rightarrow \infty} \alpha_{n}=0$;

(ii) $\sum_{n=0}^{\infty} \alpha_{n}=\infty$ or equivalently $\prod_{n=0}^{\infty}\left(1-\alpha_{n}\right)=0$;

(iii) $\lim _{n \rightarrow \infty} \frac{\alpha_{n}-\alpha_{n-1}}{\alpha_{n}}=0$.

Then the sequence $\left\{x_{n}\right\}$ generated by

$$
x_{n+1}:=\alpha_{n} u+\left(1-\alpha_{n}\right) S x_{n}, n=0,1,2, \ldots,
$$

converges strongly to a fixed point of $S$.

Our main purpose in this paper is to prove, under appropriate conditions on $K$ and $T$, that a sequence defined iteratively by: $z_{0} \in K$,

$$
z_{n+1}:=\left(1-\frac{t_{n}}{k_{n}}\right) u+\frac{t_{n}}{k_{n}} T^{n} z_{n}, n=0,1,2, \ldots,
$$

converges strongly to a fixed point of the asymptotically nonexpansive mapping $T$.

\section{Preliminaries}

Let $S:=\{x \in E:\|x\|=1\}$ denote the unit sphere of the Banach space $E$. $E$ is said to have a Gâteaux differentiable norm if the limit

$$
\lim _{n \rightarrow \infty} \frac{\|x+t y\|-\|x\|}{t}
$$

exists for each $x, y \in S$, and we call $E$ smooth; and $E$ is said to have a uniformly Gâteaux differentiable norm if for each $y \in S$ the limit is attained uniformly for $x \in S$. Furthermore, $E$ is said to be uniformly smooth if the limit exists uniformly for $(x, y) \in S \times S$. It is known that if $E$ is smooth, then any duality mapping on $E$ is single-valued, and if $E$ has a uniformly Gâteaux differentiable norm, then the duality mapping is norm-to-weak* continuous (see, e.g., [6]). Let $K$ be a nonempty closed convex and bounded subset of the Banach space $E$, and let the diameter of $K$ be defined by $d(K):=\sup \{\|x-y\|: x, y \in K\}$. For each $x \in K$, let $r(x, K):=\sup \{\|x-y\|: y \in K\}$ and let $r(K):=\inf \{r(x, K): x \in K\}$ denote the Chebyshev radius of $K$ relative to itself. The normal structure coefficient $N(E)$ of $E$ (cf. [2]) is defined by

$N(E):=\inf \left\{\frac{d(K)}{r(K)}: K\right.$ is a closed convex and bounded subset of $E$ with $\left.d(K)>0\right\}$.

A space $E$ such that $N(E)>1$ is said to have uniform normal structure. It is known that every space with a uniform normal structure is reflexive, and that all uniformly convex and uniformly smooth Banach spaces have uniform normal structure (see, e.g., 1] or [11]).

We shall let LIM be a Banach limit. Recall that LIM $\in\left(\ell^{\infty}\right)^{*}$ such that $\|\operatorname{LIM}\|=$ 1, $\liminf _{n \rightarrow \infty} a_{n} \leq \operatorname{LIM}_{n} a_{n} \leq \limsup _{n \rightarrow \infty} a_{n}$, and $\operatorname{LIM}_{n} a_{n}=\operatorname{LIM}_{n} a_{n+1}$ for all $\left\{a_{n}\right\}_{n} \in \ell^{\infty}$.

The following lemmas will be needed in the sequel.

Lemma 2.1. Let $E$ be an arbitrary real Banach space. Then

$$
\|x+y\|^{2} \leq\|x\|^{2}+2\langle y, j(x+y)\rangle
$$

$\forall x, y \in E$ and $\forall j(x+y) \in J(x+y)$. 
Lemma 2.2 ([1]). Suppose $X$ is a Banach space with uniformly normal structure, $C$ is a nonempty bounded subset of $X$, and $T: C \longrightarrow C$ is a uniformly $k$ Lipschitzian mapping with $k<N(X)^{\frac{1}{2}}$. Suppose also that there exists a nonempty bounded closed convex subset $A$ of $C$ with the following property $(\mathrm{P})$ :

$$
x \in A \text { implies } \omega_{w}(x) \subset A \text {, }
$$

where $\omega_{w}(x)$ is the weak $\omega$-limit set of $T$ at $x$, i.e., the set

$$
\left\{y \in X: y=\text { weak- } \lim _{j} T^{n_{j}} x \text { for some } n_{j} \rightarrow \infty\right\} .
$$

Then $T$ has a fixed point in $A$.

Lemma 2.3 (see, e.g., [17). Let $\left\{a_{n}\right\}$ be a sequence of nonnegative real numbers satisfying the following relation:

$$
a_{n+1} \leq\left(1-\alpha_{n}\right) a_{n}+\sigma_{n}, n \geq 0,
$$

where (i) $0<\alpha_{n}<1$; (ii) $\sum_{n=1}^{\infty} \alpha_{n}=\infty$. Suppose, either $(a) \sigma_{n}=o\left(\alpha_{n}\right)$, or (b) $\lim \sup \sigma_{n} \leq 0$. Then $a_{n} \rightarrow 0$ as $n \rightarrow \infty$.

\section{MAin RESUlts}

In the sequel $F(T)$ will denote the fixed point set of $T$.

Theorem 3.1. Let $E$ be a real Banach space with a uniformly Gâteaux differentiable norm possessing uniform normal structure, $K$ a nonempty closed convex and bounded subset of $E, T: K \longrightarrow K$ an asymptotically nonexpansive mapping with sequence $\left\{k_{n}\right\}_{n} \subset[1, \infty)$. Let $u \in K$ be fixed, $\left\{t_{n}\right\}_{n} \subset(0,1)$ be such that $\lim _{n \rightarrow \infty} t_{n}=1$, and $\lim _{n \rightarrow \infty} \frac{k_{n}-1}{k_{n}-t_{n}}=0$. Then,

(i) for each integer $n \geq 0$, there is a unique $x_{n} \in K$ such that

$$
x_{n}=\left(1-\frac{t_{n}}{k_{n}}\right) u+\frac{t_{n}}{k_{n}} T^{n} x_{n} ;
$$

and if, in addition, $\lim _{n \rightarrow \infty}\left\|x_{n}-T x_{n}\right\|=0$, then,

(ii) the sequence $\left\{x_{n}\right\}_{n}$ converges strongly to a fixed point of $T$.

Remark 1. We remark that Theorem 2 of [11] (cited here as Theorem LX) actually works in a Banach space that has a uniformly Gâteaux differentiable norm and has a uniformly normal structure. Consequently, following the lines of the proof of Theorem LX, Theorem 3.1 is easily proved.

Remark 2. Lim and Xu [11 have shown that a sequence $\left\{t_{n}\right\}_{n} \subset(0,1)$ satisfying the condition of Theorem 3.1 always exists. They gave, for example, $t_{n}:=$ $\min \left\{1-\left(k_{n}-1\right)^{\frac{1}{2}}, 1-n^{-1}\right\}$.

Corollary 3.2. Let $E$ be a real reflexive Banach space with a uniformly Gâteaux differentiable norm, $K$ a nonempty closed convex and bounded subset of $E, T$ : $K \longrightarrow K$ a completely continuous, asymptotically nonexpansive mapping with sequence $\left\{k_{n}\right\}_{n} \subset[1, \infty)$. Let $u \in K$ be fixed, $\left\{t_{n}\right\}_{n} \subset(0,1)$ be such that $\lim _{n \rightarrow \infty} t_{n}=1$, 
and $\lim _{n \rightarrow \infty} \frac{k_{n}-1}{k_{n}-t_{n}}=0$. Then,

(i) for each integer $n \geq 0$, there is a unique $x_{n} \in K$ such that

$$
x_{n}=\left(1-\frac{t_{n}}{k_{n}}\right) u+\frac{t_{n}}{k_{n}} T^{n} x_{n}
$$

and if, in addition, $\lim _{n \rightarrow \infty}\left\|x_{n}-T x_{n}\right\|=0$, then,

(ii) the sequence $\left\{x_{n}\right\}_{n}$ converges strongly to a fixed point of $T$.

Proof. For each integer $n \geq 0$, the mapping $f_{n}: K \longrightarrow K$ defined for each $x \in K$ by $f_{n} x:=\left(1-\frac{t_{n}}{k_{n}}\right) u+\frac{t_{n}}{k_{n}} T^{n} x$ is a contraction. It follows that there exists a unique $x_{n} \in K$ such that $f_{n} x_{n}=x_{n}$. Since $T$ is completely continuous there is a subsequence $\left\{T x_{n_{i}}\right\}_{i}$ of $\left\{T x_{n}\right\}_{n}$ that converges strongly to some $y^{*} \in K$, and since $\left\|x_{n_{i}}-T x_{n_{i}}\right\| \longrightarrow 0$ as $i \rightarrow \infty$, we have that $y^{*}=T y^{*}$. The rest of the proof follows as in the proof of Theorem 3.1.

Theorem 3.3. Let $E$ be a real Banach space with a uniformly Gâteaux differentiable norm possessing uniform normal structure, $K$ be a nonempty closed convex and bounded subset of $E, T: K \longrightarrow K$ be an asymptotically nonexpansive mapping with sequence $\left\{k_{n}\right\}_{n} \subset[1, \infty)$. Let $u \in K$ be fixed, $\left\{t_{n}\right\}_{n} \subset(0,1)$ be such that $\lim _{n \rightarrow \infty} t_{n}=1, t_{n} k_{n}<1$, and $\lim _{n \rightarrow \infty} \frac{k_{n}-1}{k_{n}-t_{n}}=0$. Define the sequence $\left\{z_{n}\right\}_{n}$ iteratively by $z_{0} \in K$,

$$
z_{n+1}=\left(1-\frac{t_{n}}{k_{n}}\right) u+\frac{t_{n}}{k_{n}} T^{n} z_{n}, n=0,1,2, \ldots
$$

Then,

(i) for each integer $n \geq 0$, there is a unique $x_{n} \in K$ such that

$$
x_{n}=\left(1-\frac{t_{n}}{k_{n}}\right) u+\frac{t_{n}}{k_{n}} T^{n} x_{n} ;
$$

and if, in addition, $\lim _{n \rightarrow \infty}\left\|x_{n}-T x_{n}\right\|=0, \lim _{n \rightarrow \infty}\left\|z_{n}-T z_{n}\right\|=0$, then

(ii) $\left\{z_{n}\right\}_{n}$ converges strongly to a fixed point of $T$.

Proof. From (5), $x_{m}-z_{n}=\left(1-\frac{t_{m}}{k_{m}}\right)\left(u-z_{n}\right)+\frac{t_{m}}{k_{m}}\left(T^{m} x_{m}-z_{n}\right)$. Applying inequality (3), we estimate as follows:

$$
\begin{aligned}
\| x_{m}- & z_{n}\left\|^{2} \leq \frac{t_{m}^{2}}{k_{m}^{2}}\right\| T^{m} x_{m}-z_{n} \|^{2}+2\left(1-\frac{t_{m}}{k_{m}}\right)\left\langle u-z_{n}, j\left(x_{m}-z_{n}\right)\right\rangle \\
\leq & \frac{t_{m}^{2}}{k_{m}^{2}}\left[\left\|T^{m} x_{m}-T^{m} z_{n}\right\|+\left\|T^{m} z_{n}-z_{n}\right\|\right]^{2} \\
& +2\left(1-\frac{t_{m}}{k_{m}}\right)\left[\left\langle u-x_{m}, j\left(x_{m}-z_{n}\right)\right\rangle+\left\|x_{m}-z_{n}\right\|^{2}\right] \\
\leq & \frac{t_{m}^{2}}{k_{m}^{2}}\left[k_{m}\left\|x_{m}-z_{n}\right\|+\left\|T^{m} z_{n}-z_{n}\right\|\right]^{2} \\
& +2\left(1-\frac{t_{m}}{k_{m}}\right)\left[\left\langle u-x_{m}, j\left(x_{m}-z_{n}\right)\right\rangle+k_{m}^{2}\left\|x_{m}-z_{n}\right\|^{2}\right] \\
= & \frac{t_{m}^{2}}{k_{m}^{2}}\left[k_{m}^{2}\left\|x_{m}-z_{n}\right\|^{2}+2 k_{m}\left\|x_{m}-z_{n}\right\|\left\|T^{m} z_{n}-z_{n}\right\|+\left\|T^{m} z_{n}-z_{n}\right\|^{2}\right] \\
& +2\left(1-\frac{t_{m}}{k_{m}}\right)\left[\left\langle u-x_{m}, j\left(x_{m}-z_{n}\right)\right\rangle+k_{m}^{2}\left\|x_{m}-z_{n}\right\|^{2}\right]
\end{aligned}
$$




$$
\begin{aligned}
= & \left(1-\left(1-\frac{t_{m}}{k_{m}}\right)\right)^{2} k_{m}^{2}\left\|x_{m}-z_{n}\right\|^{2}+\left\|T^{m} z_{n}-z_{n}\right\|\left[2 k_{m}\left\|x_{m}-z_{n}\right\|\right. \\
& \left.+\left\|T^{m} z_{n}-z_{n}\right\|\right]+2\left(1-\frac{t_{m}}{k_{m}}\right)\left[\left\langle u-x_{m}, j\left(x_{m}-z_{n}\right)\right\rangle+k_{m}^{2}\left\|x_{m}-z_{n}\right\|^{2}\right] \\
\leq & \left(1+\left(1-\frac{t_{m}}{k_{m}}\right)^{2}\right) k_{m}^{2}\left\|x_{m}-z_{n}\right\|^{2}+\left\|T^{m} z_{n}-z_{n}\right\| M \\
& +2\left(1-\frac{t_{m}}{k_{m}}\right)\left\langle u-x_{m}, j\left(x_{m}-z_{n}\right)\right\rangle,
\end{aligned}
$$

for some constant $M>0$. It follows that

$$
\begin{aligned}
\limsup _{n \rightarrow \infty}\left\langle u-x_{m}, j\left(z_{n}-x_{m}\right)\right\rangle \leq & \frac{\left[k_{m}^{2}-1+k_{m}^{2}\left(1-\frac{t_{m}}{k_{m}}\right)^{2}\right]}{2\left(1-\frac{t_{m}}{k_{m}}\right)} \limsup _{n \rightarrow \infty}\left\|x_{m}-z_{n}\right\|, \\
& +\limsup _{n \rightarrow \infty} \frac{M\left\|z_{n}-T^{m} z_{n}\right\|}{\left(1-\frac{t_{m}}{k_{m}}\right)}
\end{aligned}
$$

Observe that $\frac{\left[k_{m}^{2}-1+k_{m}^{2}\left(1-\frac{t_{m}}{k_{m}}\right)^{2}\right]}{2\left(1-\frac{t_{m}}{k_{m}}\right)}=\frac{k_{m}\left(k_{m}+1\right)}{2}\left[\frac{k_{m}-1}{k_{m}-t_{m}}\right]+\frac{k_{m}^{2}}{2}\left(1-\frac{t_{m}}{k_{m}}\right) \rightarrow 0$ as $m \rightarrow$ $\infty$. Since $\left\{z_{n}\right\}$ and $\left\{x_{m}\right\}$ are bounded, $\left\{T^{m} z_{n}\right\}$ is bounded and $\left\|z_{n}-T z_{n}\right\| \rightarrow 0$ as $n \rightarrow \infty$, it follows from the last inequality that

$$
\limsup _{m \rightarrow \infty} \limsup _{n \rightarrow \infty}\left\langle u-x_{m}, j\left(z_{n}-x_{m}\right)\right\rangle \leq 0 .
$$

But by Theorem 3.1 we have that $x_{m} \rightarrow x^{*} \in F(T)$ as $m \rightarrow \infty$. Moreover, $j$ is norm to weak* uniformly continuous on bounded sets. Therefore, there exists $N>0$ such that

$$
\left|\left\langle x^{*}-x_{m}, j\left(z_{n}-x_{m}\right)\right\rangle\right|<\frac{\epsilon}{2} \text { and }\left|\left\langle u-x^{*}, j\left(z_{n}-x_{m}\right)-j\left(z_{n}-x^{*}\right)\right\rangle\right|<\frac{\epsilon}{2},
$$

for all $n, m \geq N$. This implies

$$
\begin{aligned}
& \left|\left\langle u-x_{m}, j\left(z_{n}-x_{m}\right)\right\rangle-\left\langle u-x^{*}, j\left(z_{n}-x^{*}\right)\right\rangle\right| \\
\leq & \left|\left\langle u-x_{m}, j\left(z_{n}-x_{m}\right)\right\rangle-\left\langle u-x^{*}, j\left(z_{n}-x_{m}\right)\right\rangle\right| \\
& +\left|\left\langle u-x^{*}, j\left(z_{n}-x_{m}\right)\right\rangle-\left\langle u-x^{*}, j\left(z_{n}-x^{*}\right)\right\rangle\right| \\
= & \left|\left\langle x^{*}-x_{m}, j\left(z_{n}-x_{m}\right)\right\rangle\right|+\left|\left\langle u-x^{*}, j\left(z_{n}-x_{m}\right)-j\left(z_{n}-x^{*}\right)\right\rangle\right| \\
\leq & \epsilon \text { for all } n, m \geq N .
\end{aligned}
$$

Thus, from (6) and (7),

$$
\limsup _{n \rightarrow \infty}\left\langle u-x^{*}, j\left(z_{n}-x^{*}\right)\right\rangle \leq \limsup _{m \rightarrow \infty} \limsup _{n \rightarrow \infty}\left\langle u-x_{m}, j\left(z_{n}-x_{m}\right)\right\rangle+\epsilon \leq \epsilon .
$$

Since $\epsilon>0$ is arbitrary, we obtain that

$$
\limsup _{n \rightarrow \infty}\left\langle u-x^{*}, j\left(z_{n}-x^{*}\right)\right\rangle \leq 0 .
$$

Now from the iteration procedure (4) and inequality (3) we have that

$$
\begin{aligned}
\left\|z_{n+1}-x^{*}\right\|^{2} & \leq \frac{t_{n}^{2}}{k_{n}^{2}}\left\|T^{n} z_{n}-x^{*}\right\|^{2}+2\left(1-\frac{t_{n}}{k_{n}}\right)\left\langle u-x^{*}, j\left(z_{n+1}-x^{*}\right)\right\rangle \\
& \leq \frac{t_{n}}{k_{n}}\left\|z_{n}-x^{*}\right\|^{2}+2\left(1-\frac{t_{n}}{k_{n}}\right)\left\langle u-x^{*}, j\left(z_{n+1}-x^{*}\right)\right\rangle, \\
& \leq\left(1-\alpha_{n}\right)\left\|z_{n}-x^{*}\right\|^{2}+2 \alpha_{n} \beta_{n},
\end{aligned}
$$


where $\alpha_{n}:=\left(1-\frac{t_{n}}{k_{n}}\right)$. So $\beta_{n}:=\left\langle u-x^{*}, j\left(z_{n+1}-x^{*}\right)\right\rangle$, so that $\lim \sup \alpha_{n} \beta_{n} \leq 0$. It now follows from Lemma 2.3 that $z_{n} \rightarrow x^{*}$ as $n \rightarrow \infty$, completing the proof.

Corollary 3.4. Let $E$ be a real reflexive Banach space with a uniformly Gâteaux differentiable norm, let $K$ be a nonempty closed convex and bounded subset of $E$, and let $T: K \longrightarrow K$ be a completely continuous asymptotically nonexpansive mapping with sequence $\left\{k_{n}\right\}_{n} \subset[1, \infty)$. Let $u \in K$ be fixed, $\left\{t_{n}\right\}_{n} \subset(0,1)$ be such that $\lim _{n \rightarrow \infty} t_{n}=1, t_{n} k_{n}<1$ and $\lim _{n \rightarrow \infty} \frac{k_{n}-1}{k_{n}-t_{n}}=0$. Define the sequence $\left\{z_{n}\right\}_{n}$ iteratively $n \rightarrow \infty$
by $z_{0} \in K$,

$$
z_{n+1}=\left(1-\frac{t_{n}}{k_{n}}\right) u+\frac{t_{n}}{k_{n}} T^{n} z_{n}, n=0,1,2, \ldots
$$

Then,

(i) for each integer $n \geq 0$, there is a unique $x_{n} \in K$ such that

$$
x_{n}=\left(1-\frac{t_{n}}{k_{n}}\right) u+\frac{t_{n}}{k_{n}} T^{n} x_{n} ;
$$

and if, in addition, $\lim _{n \rightarrow \infty}\left\|x_{n}-T x_{n}\right\|=0$ and $\lim _{n}\left\|z_{n}-T z_{n}\right\|=0$, then,

(ii) $\left\{z_{n}\right\}_{n}$ converges strongly to a fixed point of $T$.

Proof. As in the proof of Corollary 3.2 there exists $y^{*} \in K$ such that $T y^{*}=y^{*}$. The rest of the proof follows as in Theorem 3.3.

Remark 3. An example of a sequence $\left\{t_{n}\right\}_{n} \subset(0,1)$ satisfying the conditions of Theorem 3.3 and Corollary 3.4 is given by

$$
t_{n}:=\min \left\{k_{n}^{-1}-k_{n}^{-1}\left(k_{n}-1\right)^{\frac{1}{2}}, 1-n^{-1}\right\}, n=0,1,2, \ldots .
$$

Remark 4. We observe that if we set $\alpha_{n}=1-\frac{t_{n}}{k_{n}}$ and $T$ is nonexpansive, in which case $k_{n} \equiv 1 \forall$ integers $n \geq 0$, then, replacing $T^{n}$ by $T$, our iteration process (21) reduces to the one studied by Xu [17]. Moreover, the boundedness of $K$ in Theorem 3.3 is no longer required. Only the boundedness of the sequence $\left\{z_{n}\right\}$ is needed, and this follows as in Xu [17]. Furthermore, in this case the condition $\lim _{n \rightarrow \infty}\left\|x_{n}-T x_{n}\right\|=0$ is satisfied. The choice $t_{n}:=\min \left\{k_{n}^{-1}-k_{n}^{-1}\left(k_{n}-1\right)^{\frac{1}{2}}, 1-n^{-1}\right\}$ reduces to $\alpha_{n}=\frac{1}{n}$, which is the natural choice that is part of the motivation of the paper of $\mathrm{Xu}$ [17. Finally, it is well known (see, e.g., 6] and 9]) that every uniformly smooth Banach space has a uniformly Gâteaux differentiable norm and possesses uniform normal structure.

\section{ACKNOWLEDGMENTS}

This work was undertaken when the third author was visiting the Abdus Salam ICTP, Trieste as an Associate. He is grateful to SIDA, Sweden and ICTP, Italy for the generous support that enabled this visit.

The authors thank the referee for very useful comments.

\section{REFERENCES}

[1] A. G. Aksoy and M. A. Khamsi; Nonstandard Methods in Fixed Point Theory, SpringerVerlag, New York, 1990. MR 91i:47073

[2] W. L. Bynum; Normal structure coefficients for Banach spaces, Pacific J. Math., 86 (2001), 427-436. MR 81m:46030 
[3] R. E. Bruck, T. Kuczumow and S. Reich; Convergence of iterates of asymptotically nonexpansive mappings in Banach spaces with the uniform Opial property, Colloquium Mathematicum, Vol. LXV Fasc. 2 (1993), 169-179. MR 94h:47106

[4] S-S. Chang; Some results for asymptotically pseudocontractive mappings and asymptotically nonexpansive mappings, Proc. Amer. Math. Soc., 129 (2000), 845-853. MR 2002b:47124

[5] C. E. Chidume; Convergence theorems for asymptotically pseudocontractive mappings, Nonlinear Analysis, TMA, 49 (2002), 1-11. MR 2002k:47109

[6] I. Cioranescu; Geometry of Banach spaces, Duality mappings and Nonlinear Problems, Kluwer Academic Publishers, Amsterdam, 1990. MR 91m:46021

[7] K. Goebel and W. A. Kirk; A fixed point theorem for asymptotically nonexpansive mappings, Proc. Amer. Math. Soc., 35 (1972), 171-174. MR 45:7552

[8] K. Goebel and W. A. Kirk; A fixed point theorem for transformations whose iterates have uniform Lipschitz constant, Studia Math., 47 (1973), 135-140. MR 49:1242

[9] K. Goebel and W. A. Kirk; Topics of Metric Fixed Point Theory, Cambridge Univ. Press, Cambridge, 1990. MR 92c:47070

[10] B. Halpern; Fixed points of nonexpansive maps, Bull. Amer. Math. Soc. 3 (1967), 957-961. MR 36:2022

[11] T. C. Lim and H. K. Xu; Fixed point theorems for asymptotically nonexpansive mappings, Nonlinear Analysis, TMA, 22 (1994), 1345-1355. MR 95i:47103

[12] N. Shioji and W. Takahashi; A strong convergence theorem for asymptotically nonexpansive mappings in Banach spaces, Arch. Math. (Basel) 72 (1999), 354-359. MR 2001b:47091

[13] N. Shioji and W. Takahashi; Strong convergence of averaged approximants for asymptotically nonexpansive mappings in Banach spaces, J. Approx. Theory 97 (1999), 53-64. MR 99m:41055

[14] J. Schu; Iterative construction of fixed points of asymptotically nonexpansive mappings, $J$. Math. Anal. Appl., 158 (1991), 407-413. MR 92d:47072

[15] J. Schu; Approximation of fixed points of asymptotically nonexpansive mappings, Proc. Amer. Math. Soc., 112(1) (1991), 143-151. MR 91h:47063

[16] K. K. Tan and H. K. Xu; Fixed point iteration process for asymptotically nonexpansive mappings, Proc. Amer. Math. Soc., 122 (1994), 733-739. MR 95a:47070

[17] H. K. Xu; Iterative algorithms for nonlinear operators, J. London Math. Soc., 66 (2002), 240-256. MR 2003e:47114

The Abdus Salam International Centre for Theoretical Physics, Trieste, Italy

E-mail address: chidume@ictp.trieste.it

Department of Mathematics, Shawnee State University, Portsmouth, Ohio 45662

E-mail address: jli@shawnee.edu

Department of Mathematics, Statistics, Computer Science, University of Port HarCourt, Port Harcourt, Nigeria

E-mail address: EpsilonAni@aol.com 